\title{
The Physiotherapist and the Treatment of Anterior Poliomyelitis
}

\author{
BY SYLVIA SOUTHERN, M.C.S.P., D.P.T.
}

\begin{abstract}
N SPITE OF THE ADVANCES in Medicine in recent years, the prevention and cure of Anterior Poliomyelitis still puzles the most brilliant brains in Medical Research.
\end{abstract}

In South Africa, where we have just experienced one of our worst epidemics, the call for a fund to finance an extensive exploration into the (1) cause, (2) channels by which infection is carried, and (3) prevention of Poliomyelitis will surely meet with a hearty response from all sections of the community. Although only a few hundreds of the population have been smitten with the disease, while thousands have been affected in England, America and Australia in recent epidemics, we must remember that the population is smaller, and therefore the damage in comparison is as great.

The need for specialised treatment is apparent from the outset. Nowhere is the necessity more marked than in Poliomyelitis, for the damage caused mentally and physically to the patient is for a long period, which may extend from three weeks to two years, and very often for the whole life of an individual. Although much can be achieved by Doctors and Nurses, the residual symptoms can only be healed by the mental and bodily activities of the patient in close co-operation with the Physiotherapist. Long periods of rest are necessary, and it is during this time that the services of a well qualified Physiotherapist are essential.

The human body was not built to remain at rest indefinitely. Activity is essential for the recovery of health, and early movement will repair wasted tissue. The skin loses its function when immobilized - it becomes scaly, rough and unhealthy. Impaired circulation affects the organs of digestion and elimination. Nutrition and metabolism are upset, and one of the main roads to reestablish health is blocked.

The mental effect of treatment is now well realised, and every hospital where Anterior Poliomyelitis is treated has its staff of Physiotherapists and Occupational or Diversional Therapists, for the danger of the stagnant body is fully reognised by many members of the Medical Profession.

If rest is an important factor in the healing of Poliomyelitis, the Public Health authorities must avail themselves of a suitable hospital where everything is available for the comfort of the patient and for the treatment of the disease. An early decision must be made as to whether hospitalisation is imperative.

There may be hesitation over this, because of the lack of knowledge as to how the disease is contracted. If hospitalisation is required, it is advisable that continuity of treatment should be carried out in the one Institution for the first six to twelve months, as it is usual for all stages of paralysis to have manifested themselves during that time.

All wards, bath rooms, treatment rooms and workshops should be on the ground floor. Every requirement for the immediate comfort of the patient should be eas'ly obtainable, whatever it may be, from an Iron Lung to a Monkey Chain or Fracture Board or Sandbag. The wards should be spacious and airy, with well regulated heat if necessary. They should be painted in soothing colours, as the prevailing atmosphere required is one of calm, hope and freedom.

Nurses and Physiotherapists should be chosen for their mental affect on the patients. Let them wear coloured overalls-white so often strikes holy terror in a nervous child. They must be sympathetic and exude optimism. A refreshing personality gives the patient a feeling of comfort which results in confidence, thereby inducing relaxation. There is a general feeling that "all is well." It is also very desirable that Nurses and Physiotherapists have a thorough knowledge of Orthopaedic Nursing.

One Nurse to every four patients is ideal, and one Nursing Sister and one Physiotherapist should be in charge of every ward. Co-operation between Doctors, Physiotherapists, Sisters and Nurses is the order of the day.

We now consider the bed on which the sick patient must be rested. It should be flat and firm, with a mattress made in sections to ensure clean and easy nursing. When no complications are likely to occur the patient is placed flat on the bed with the body in strict alignment. The legs and arms must be arranged in a comfortable, relaxed position; and an adaptable footboard placed at the 
foot of the bed to give the patient a firm support to push himself on, and to keep the feet dorsi flexed. This board will also prevent pressure of the bed clothes. The patient must not be constricted in any way. By constant supervision limbs can be kept in a good natural position by means of sandbags and small pillows.

The types of Polio are various, but nearly all sufferers are affected mentally in some way or another. This calls for a good personality in those who have anything to do for these cases. Each case requires individual treatment. The types are differentiated by the region of the involvment of the spinal cord and brain. Thus we have the Bulbar Poliomyelitis, the Encephalitic Poliomyelitis, the Paralytic Poliomyelitis and the Abortive. Fortunately the Abortive type is the most usual. The patient is ill for a short duration, and symptoms may last for a few hours or a few days. Vomiting and headache are the chief characteristics of this type. There may be some spasm, usually in the lumbar region, sometimes in the arms and legs. Daily vigilance on the part of the Physiotherapist is required for the early detection of weakness in muscles, groups of muscles and limbs, but on no account should the patient be worried or wearied. This type, when willing and eager to recover, responds quickly to muscle re-education, and the recovery is so rapid as to be miraculous. At a first treatment the patient appears unable to move the whole of one limb, but when fear is overcome and movement is evident the apparent loss of function is quickly restored. It is understood that the patient is resting in bed and that no weight bearing exercises are allowed until the patient is sufficiently recovered and is able to perform a complete series of movements ivolving all joints while in the recumbent position. The next progression is to sit and then stand. This type is usually fit to return home after the three weeks allowed for the infectious period.

When there is spasm, this can remain for some time, and in some cases for as long as six weeks or two months. It can be relieved in a great measure by hot packs, hot cradles, hot saline baths and sometimes by very gentle, soothing stroking of the spine, back muscles and gluteii muscles. The back stroking has a very good psychological effect on the patient, who is very often irritable and despondent.

The Bulbar type of Poliomyelitis is the most serious, as it affects the nose, throat and respiratory muscles. The patient is of ten unable to swallow, and breathing is enfeebled. These children are very ill, and few recover, but the Iron Lung treatment combined with various medical treatments and super human nursing has brought these sufferers from the jaws of death - sometimes, alas, with paralysis of large groups of muscles of arms, legs and back. As soon as these patients are sufficiently well to remain for a short period out of the Iron Lung, it is amazing to see what results can be obtained by the Physiotherapist. She is asked to give a few breathing exercises daily, for a few minutes at the commencement, and gradually progresses to gentle massage to all limbs, and gentle movements of all joints within the inner range of movement. Great care is exercised not to exhaust the patient. The treatment and progression must of necessity be very slow and it is only when they have recovered sufficiently to be able to leave the Lung for all time that treatment can be progressed to hydrotherapy, electrotherapy and the like. Too of ten recovery of muscles is retarded or even frustrated by too much enthusiasm on the part of the Physiotherapist to bring about a miraculous and spectacular result. One or two gentle faradic stimulii are beneficial to a muscle recovering from paralysis, but overwork will kill it. The psychological effect is good.

The Encephalitic type of Poliomyelitis will make a total recovery after being very seriously ill.

The Paralytic type often has a group of muscles or one particular muscle paralysed. The most common groups are the muscles of the shoulder girdle. The Quadriceps Extensors, the Anterior Tibial group, the Gluteii and Hamstrings. There are cases where one muscle only is paralysed, such as the Tibialis Anticus, which is the most usual one to be picked out. Sometimes it may be the Peroneus Longus.

The aims of the Physiotherapist are therefore to relieve spasm, induce relaxation, prevent deformity and restore function.

The work of the Physiotherapist can usually commence on the tenth or fourteenth day, according to the condition of the patient -both children and adults. The treatment must be of a high order, for any tendency to roughness or undue pressure on tender and painful tissues may result in total paralysis, where recovery may otherwise have been obtained. The moral value of early treatment cannot be over emphasised. 